Прогнозирование тяжести

коронарного атеросклероза

у пациентов кардиологического профиля

\author{
Ю.Н. Федулаев ${ }^{\varpi 1}$, И.В. Макарова' ${ }^{1}$ Т.В. Пинчук' , С.Э. Аракелов ${ }^{2,3}$, И.Ю. Титова ${ }^{2}$ \\ 'ФГАОУ ВО «Российский национальный исследовательский медицинский университет им. Н.И. Пирогова» Минздрава России, Москва, Россия; \\ 2ГБУЗ «Городская клиническая больница №13» Департамента здравоохранения г. Москвы, Москва, Россия; \\ ЗФГАОУ ВО «Российский университет дружбы народов», Москва, Россия
}

\begin{abstract}
Аннотация
Обоснование. Коронарный атеросклероз представляет собой непрерывно протекающий патологический процесс, проявления которого варьируют от бессимптомных форм до стенокардии, инфаркта миокарда и внезапной смерти. Раннее выявление лиц с высоким риском выраженного атеросклероза коронарного русла позволит осуществить адекватные диагностические и лечебные мероприятия, направленные на предупреждение сердечно-сосудистых осложнений.

Цель. Построить модель, позволяющую определить вероятность значимого коронарного атеросклероза у кардиологических больных.

Материал и методы. В исследование включены 116 пациентов кардиологического профиля с разной степенью тяжести атеросклероза по данным коронарографии: 1-я группа - стеноз, занимающий $\geqslant 70 \%$ просвета сосуда ( $250 \%$ при поражении ствола), 2-я группа - менее выраженный атеросклеротический процесс. Всем участникам были выполнены электрокардиография (ЭКГ) в состоянии покоя и холтеровское мониторирование ЭКГ. Во всех случаях определялось наличие патологических зубцов $Q$, оценивались качественные и количественные характеристики желудочковых нарушений ритма и фрагментации QRS-комплексов. У лиц с синусовым ритмом дополнительно рассчитывались турбулентность сердечного ритма, микроальтернация зубца $T$, дисперсии интервала QT и QTc на максимальных и минимальных значениях частоты сердечных сокращений.

Результаты. В прогностическую модель вошли следующие ЭКГ-переменные: турбулентность сердечного ритма, патологические зубцы Q, дисперсия QTc на максимальных значениях частоты сердечных сокращений и фрагментация QRS в отведениях I, AVL, V6. Bce показатели имели прямую связь с вероятностью развития выраженного атеросклероза коронарного русла. Разработанная модель учитывала $71 \%$ факторов,

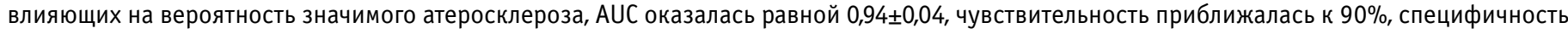
составила $94,4 \%$.

Заключение. Комплексная оценка данных ЭКГ-методов исследования позволяет своевременно идентифицировать группу высокого риска тяжелого коронарного атеросклероза среди пациентов кардиологического профиля.
\end{abstract}

Ключевые слова: электрокардиография, холтеровское мониторирование, атеросклероз, дисперсия интервала QT, турбулентность сердечного ритма, фрагментация QRS-комплекса

Для цитирования: Федулаев Ю.Н., Макарова И.В., Пинчук Т.В., Аракелов С.Э., Титова И.Ю. Прогнозирование тяжести коронарного атеросклероза у пациентов кардиологического профиля. CardioСоматика. 2021; 12 (1): 11-14. DOI: 10.26442/22217185.2021.1.200766

\section{Введение}

Атеросклероз коронарных артерий представляет собой длительный, непрерывно протекающий патологический процесс, развитию которого способствуют как генетические, так и потенциально модифицируемые факторы риска. Проявления атеросклероза варьируют от бессимптомных форм до стенокар-

\section{Информация об авторах / Information about the authors}

\begin{abstract}
Федулаев Юрий Николаевич - д-р мед. наук, проф., зав. каф. факультетской терапии педиатрического фак-та ФГАОУ ВО «РНИМУ им. Н.И. Пирогова». E-mail: kuwert@yandex.ru; ORCID: 0000-0003-4040-2971
\end{abstract}

Макарова Ирина Владимировна - ассистент каф. факультетской терапии педиатрического фак-та ФГАОУ ВО «РНИМУ им. Н.И. Пирогова».

ORCID: 0000-0001-5127-1300

Пинчук Татьяна Витальевна - канд. мед. наук, доц. каф. факультетской терапии педиатрического фак-та ФГАОУ ВО «РНИМУ им. Н.И. Пирогова». ORCID: 0000-0002-7877-4407

Аракелов Сергей Эрнестович - д-р мед. наук, глав. врач ГБУЗ ГКБ №13, зав. каф. семейной медицины с курсом паллиативной медицинской помощи ФГАОУ ВО РУДН. ОRСID: 0000-0003-3911-8543

Титова Ирина Юрьевна - зам. глав. врача по мед. части ГБУЗ ГКБ №13. ORCID: 0000-0001-7056-0634 дии, инфаркта миокарда и внезапной смерти [1]. Современные визуализирующие методы - коронароангиография, оптическая когерентная томография, мультиспиральная компьютерная томография (МСКТ), радиоизотопные методы - позволяют оценить выраженность атеросклеротического процесса у конкретного
Yuri N. Fedulaev - D. Sci. (Med.), Prof., Pirogov Russian National Research Medical University. E-mail: kuwert@yandex.ru; ORCID: 0000-0003-4040-2971

Irina V. Makarova - Assistant, Pirogov Russian National Research Medical University. ORCID: 0000-0001-5127-1300

Tatiana V. Pinchuk - Cand. Sci. (Med.), Pirogov Russian National Research Medical University. ORCID: 0000-0002-7877-4407

Sergey E. Arakelov - D. Sci. (Med.), City Clinical Hospital №13, People's Friendship University of Russia. ORCID: 0000-0003-3911-8543

Irina Yu. Titova - Deputy Chief of City Clinical Hospital №13. ORCID: 0000-0001-7056-0634 


\title{
Predicting the coronary atherosclerosis severity in cardiac patients
}

\author{
Yuri N. Fedulaev', Irina V. Makarova', Tatiana V. Pinchuk', Sergey E. Arakelov, ${ }^{2,}$ Irina Yu. Titova ${ }^{2}$ \\ ${ }^{1}$ Pirogov Russian National Research Medical University, Moscow, Russia; \\ ${ }^{2}$ City Clinical Hospital №13, Moscow, Russia; \\ ${ }^{3}$ People's Friendship University of Russia, Moscow, Russia
}

\begin{abstract}
Background. Coronary atherosclerosis is an ongoing pathological process, varying from asymptomatic forms to angina pectoris, myocardial infarction and even sudden cardiac death. Early identification of persons with an increased risk of the severe atherosclerosis will promote adequate diagnostic and therapeutic measures to prevent cardiovascular complications.

Aim. To make a prognostic model determining the probability of a severe coronary atherosclerosis in cardiac patients.

Material and methods. The actual study included 116 patients of cardiology departments with various degree of coronary atherosclerosis measured by coronary angiography: group I $\geqslant 70 \%$ coronary stenosis ( $\geqslant 50 \%$ in case ofleft main coronary artery), group II - those withless severe atherosclerotic process. All patients underwent electrocardiography (ECG) at rest and Holter monitoring. Pathological $Q$-waves, qualitative and quantitative characteristics of ventricular extrasystoles as well as QRS-fragmentation were assessed in all cases. In individuals having sinus rhythm, heart rate turbulence (HRT), T-wave alternans and QT, QTC dispersion on maximum and minimum heart rate were additionally calculated. Results. The prognostic model included the following ECG-markers: HRT, pathological Q-waves, QTC dispersion on maximum heart rate and QRS-fragmentation in lateral leads (I, AVL, V6). All parameters have demonstrated a direct relationship with thelikelihood of severe coronary atherosclerosis. The current model took into account $71 \%$ of the factors influencing significant atherosclerosis, $A U C=0.94 \pm 0.04$, the sensitivity and the specificity were 90.0 and $94.4 \%$ respectively.

Conclusion. A comprehensive assessment of the ECG data helps to identify the group with an increased risk of severe coronary atherosclerosis among cardiac patients.
\end{abstract}

Keywords: electrocardiography, Holter monitoring, atherosclerosis, QT dispersion, heart rate turbulence, QRS-fragmentation For citation: Fedulaev YuN, Makarova IV, Pinchuk TV, Arakelov SE, Titova IYu. Predicting the coronary atherosclerosis severity in cardiac patients. Cardiosomatics. 2021; 12 (1): 11-14. DOI: 10.26442/22217185.2021.1.200766

пациента, а немедикаментозные (диета, физическая активность) и медикаментозные (статины, ингибиторы PCSK9) мероприятия замедлить его прогрессирование [2]. Однако на сегодняшний день рутинное проведение указанных исследований в популяции больных с подозреваемой ишемической болезнью сердца (ИБС) не обосновано [3]. Необходимость дифференцированного подхода к обследованию пациентов с жалобами на стенокардию (или ее эквиваленты) не вызывает сомнений. Один из путей решения данной задачи - использование шкалы оценки предтестовой вероятности (ПТВ) ИБС. Так, низкая ПТВ делает диагноз ИБС маловероятным и в большинстве случаев позволяет отказаться от дальнейших инструментальных методов диагностики. И, наоборот, высокая клиническая вероятность ИБС, как правило, указывает на необходимость проведения коронароангиографии. Помимо ПТВ, дополнительно могут быть оценены факторы сердечно-сосудистого риска, результаты электрокардиографии (ЭКГ), индекс Агатстона и другие показатели. Необходимо отметить, что, несмотря на свою простоту, шкала ПТВ ИБС сопряжена с рядом ограничений. В частности, в нее не включены пациенты с неангинозными жалобами (перебои в работе сердца/неритмичное сердцебиение и т.д.), а также бессимптомные больные. Важность своевременной диагностики и лечения у последних объясняется тем, что почти в 50\% случаев первым проявлением ИБС становится инфаркт миокарда или внезапная смерть [4]. С другой стороны, сохраняется потребность в проведении дополнительных (в том числе дорогостоящих и сопряженных с рядом осложнений) инструментальных исследований, подтверждающих наличие ишемии миокарда, у лиц с промежуточной ПТВ ИБС. Указанные факторы подчеркивают необходимость разработки модели, позволяющей на основании доступных и безопасных параметров осуществлять оценку целесообразности проведения коронароангиографии у пациентов с различными проявлениями ИБС (в том числе с безболевой ишемией миокарда).
Цель - построить модель, позволяющую определить вероятность выраженного атеросклероза коронарных артерий у пациентов кардиологического профиля.

\section{Материал и методы}

В проспективное исследование включены 116 пациентов C сердечно-сосудистой патологией, проходивших обследование в ГБУЗ ГКБ №13 в 2017-2020 гг. Всем участникам, согласившимся принять участие в исследовании и подписавшим информированное добровольное согласие, проведены: ЭКГ покоя, холтеровское мониторирование ЭКГ, эхокардиография и коронароангиография. Пациенты с реваскуляризацией в анамнезе, а также с инфарктом миокарда и/или нарушением мозгового кровообращения, зафиксированным в течение 2 последних месяца, не включались в актуальное исследование. Протокол исследования составлен в соответствии с ключевыми положениями Хельсинкской декларации и получил одобрение локального этического комитета. Основываясь на данных коронароангиографии, мы разделили пациентов на 2 группы, сопоставимые по возрасту и полу: со значимым атеросклерозом (стеноз коронарной артерии $\geqslant 70 \%$ или $\geqslant 50 \%$ при поражении ствола) - 1-я группа и без значимого атеросклероза - 2-я группа. У всех участников оценены следующие показатели стандартной ЭКГ: патологические зубцы $Q$ и фрагментация $Q R S$-комплексов. Фрагментация вычислялась в соответствии с критериями, указанными в таблице $[5,6]$. Оценивалось количество отведений с фрагментированными комплексами, а также локализация фрагментации.

В ходе 12-канального холтеровского мониторирования ЭКГ всем пациентам, имевшим синусовый ритм на момент обследования, помимо базовых показателей дополнительно определены микроальтернация зубца Т (МАТ) временным методом, дисперсии интервалов QT и QTc на максимальной и минимальной частоте сердечных сокращений - ЧСС (по формуле Базетта при нормосистолии и по формуле 


\begin{tabular}{|l|c|c|}
\hline \multicolumn{1}{|c|}{$\begin{array}{c}\text { Критерии фрагментации } \\
\text { QRS-комплексов }\end{array}$} & $\begin{array}{c}\text { Узкие } \\
\text { комплексы }\end{array}$ & $\begin{array}{c}\text { Широкие } \\
\text { комплексы }\end{array}$ \\
\hline $\begin{array}{l}\text { Ширина комплексов базового } \\
\text { ритма, мс }\end{array}$ & $<120$ & $\geq 120$ \\
\hline Количество зазубрин зубца $R$ & $\geq 1$ & $>2$ \\
\hline Количество зазубрин зубца $S$ & $\geq 1$ & $>2$ \\
\hline Количество зубцов $R^{\prime}$ & $\geq 1$ & $>2$ \\
\hline
\end{tabular}

Фремингема при тахи-/брадикардии), а также параметры turbulence onset (T0) и turbulence slope (TS) турбулентности сердечного ритма (ТСP) $[7,8]$. Статистическая обработка материала осуществлялась с использованием программного пакета SPSS (version 26, SPSS Inc, IBM Corporation, New York). Нормальность распределения количественных переменных определялась при помощи критерия Шапиро-Уилка. Для проведения сравнительного анализа использовались U-критерий Манна-Уитни и критерий $\chi^{2}$ Пирсона. Различия считались статистически значимыми в случаях, если $p<0,05$. При построении прогностической модели применялись методы бинарной логистической регрессии и построение ROC-кривых.

\section{Результаты}

Результаты коронароангиографии представлены в виде бинарного показателя, где 0 - отсутствие значимого атеросклероза коронарных артерий (2-я группа), 1 - наличие значимого атеросклероза (1-я группа). В качестве факторных признаков рассмотрены следующие переменные: патологические зубцы $Q$, МАТ, ТСР, дисперсии интервалов QT и QTc на максимальной и минимальной чСС, а также качественные и количественные характеристики желудочковых нарушений ритма и фрагментации $Q R S$-комплексов. Полученное уравнение бинарной логистической регрессии выглядело следующим образом:

$$
\begin{gathered}
p=\frac{1}{\left(1+e^{-z}\right),} \\
\mathrm{Z}=-24+0,1 * \mathrm{X}_{\mathrm{QT}}+20,6 * \mathrm{X}_{\mathrm{TCP}}+40,8 * \mathrm{X}_{\mathrm{fQRS}}+22,9 * \mathrm{X}_{\mathrm{Q}}(1),
\end{gathered}
$$

где $p$ - вероятность значимого атеросклероза коронарных артерий, XQT - дисперсия интервала QTC на максимальных значениях ЧСС (МС), ХTСР - ТСР (0 - нет, 1 - есть), $\mathrm{X}_{\text {fORS }}$ - фрагментация QRS-комплексов в отведениях I, AVL, V6 (0 - нет, 1 - есть), $\mathrm{X}_{Q}$-патологические зубцы $Q(0-$ нет, 1 - есть). Представленная прогностическая модель статистически значимая $(p<0,001)$ и учитывает $71 \%$ факторов, влияющих на тяжесть коронарного атеросклероза. Все переменные, включенные в модель, имели прямую связь с вероятностью развития выраженного атеросклероза коронарного русла. Для определения порогового значения логистической функции мы воспользовались методом построения ROC-кривых (см. рисунок).

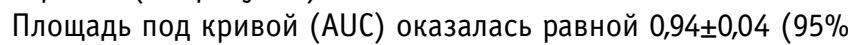
доверительный интервал 0,87-1,0). Значение логистической функции в точке cut-off составило 0,4. При $p$ равном или превышающем данное значение, прогнозировался значимый атеросклероз коронарных сосудов. При заданном пороговом значении функции р (1) чувствительность модели приблизилась к 90\%, специфичность, в свою очередь, составила $94,4 \%$.

\section{Обсуждение}

За последние десятилетия разработаны несколько моделей, прогнозирующих тяжесть ИБС. Так, в обновленных европейских рекомендациях по хроническому коронарному синдрому обосновывает-

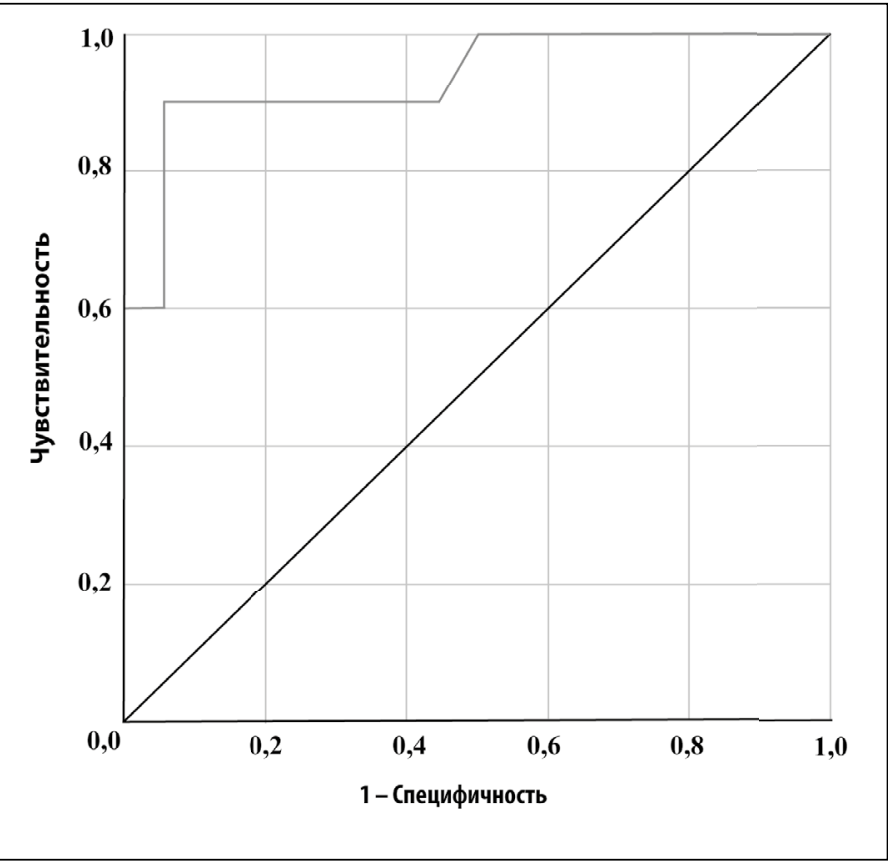

Рис. 1. ROC-кривая, описывающая зависимость тяжести атеросклероза коронарных артерий от значений логистической функции $\boldsymbol{p}$.

ся необходимость оценки анамнестических факторов, ЭКГ-данных и индекса коронарного кальция для уточнения диагноза ИБС. В то же время чувствительность ЭКГ, зарегистрированной как в состоянии покоя, так и при физической нагрузке, в диагностике ишемических изменений остается низкой. МСКТ коронарных артерий является высокочувствительным и специфичным методом (99 и 89\% соответственно) у больных с ИБС, однако сопряжена с лучевой нагрузкой и требует соответствующего оснащения и уровня подготовки специалистов $[3,9,10]$. При этом наличие коронарного кальциноза не всегда соответствует тяжелому атеросклеротическому поражению коронарных артерий по данным коронароангиографии [3]. Н.Г. Веселовской и соавт. предложена модель, основанная на клинических (повышение артериального давления, нарушение углеводного обмена) и лабораторных параметрах (уровень триглицеридов, С-реактивного белка, адипонектина и лептина), позволяющая прогнозировать наличие коронарного атеросклероза у больных с ожирением. К ограничениям исследования следует отнести малый размер выборки, а также использование в качестве эталона как коронароангиографии, так и МСКТ. Пациенты с клиническими проявлениями ИБС не включались в анализ [11]. В другой работе продемонстрирована возможность неинвазивного прогнозирования ангиографической картины при помощи нейросетей. Ключевыми предикторами атеросклероза оказались, в частности, гипертрофия левого желудочка, стенокардия, перенесенный инфаркт миокарда, а также результаты нагрузочного тестирования [12]. В еще одном исследовании указывалось на высокое диагностическое значение утолщения комплекса интима-медиа, атеросклероза сонных и бедренных артерий в выявлении коронарного атеросклероза [13]. Впрочем, среди бессимптомных лиц использование толщины интимы-медиа для скрининга ИБС в настоящее время не рекомендуется [3]. Результаты нашего исследования позволяют рассматривать ряд ЭКГ-параметров (ТСР, дисперсию интервала QTc, фрагментацию $Q R S$-комплексов в боковых отведениях и патологические зубцы Q) в качестве маркеров тяжелого атеросклероза коронарного русла. Однако сохраняется необходимость подтверждения значения вышеупомянутых маркеров в более масштабных и продолжительных исследованиях, а также изучения динамики показателей на фоне медикаментозной терапии и интервенционного лечения. 


\section{Заключение}

Комплексная оценка ЭКГ-показателей позволяет своевременно идентифицировать группу высокого риска тяжелого коронарного атеросклероза среди пациентов, обратившихся за медицинской помощью в кардиологический стационар.

Конфликт интересов. Авторы заявляют об отсутствии конфликта интересов.

Conflict of interests. The authors declare no conflict of interest.
Вклад авторов. Все авторы подтверждают соответствие своего авторства, согласно международным критериям ICMJE.

Authors' contribution. All authors confirm the compliance of their authorship according to the international ICMJE criteria.

Источник финансирования. Авторы заявляют об отсутствии внешнего финансирования при проведении исследования.

Funding source. This study was not supported by any external sources of funding.

\section{СПИСОК СОКРАЩЕНИЙ}

ИБС - ишемическая болезнь сердца

МСКТ - мультиспиральная компьютерная томография

ТСР - турбулентность сердечного ритма

ПТВ - предтестовая вероятность

чСС - частота сердечных сокращений

ЭКГ - электрокардиография

\section{Литература/References}

1. Parsons C, Agasthi P, Mookadam F, Arsanjani R. Reversal of coronary atherosclerosis: Role of life style and medical management. Trends Cardiovasc Med 2018; 28 (8): 524-31.

2. Boudoulas KD, Triposciadis F, Geleris P, Boudoulas H. Coronary Atherosclerosis: Pathophysiologic Basis for Diagnosis and Management. Prog Cardiovasc Dis 2016; 58 (6): 676-92.

3. Knuuti J, Wijns W, Achenbach S, et al. 2019 ESC guidelines for the diagnosis and management of chronic coronary syndromes. Eur Heart J 2020; 3 (41): 407-77.

4. Gutterman DD. Silent myocardial ischemia. Circ J 2009; 73 (5): 785-97.

5. Das MK, Saha C, El Masry $\mathrm{H}$, et al. Fragmented $Q R S$ on a 12-lead ECG: A predictor of mortality and cardiac events in patients with coronary artery disease. Heart Rhythm 2007; 4: 1385-92.

6. Das MK, Suradi $\mathrm{H}$, Maskoun W, et al. Fragmented wide $Q R S$ on a 12-lead ECG: a sign of myocardial scar and poor prognosis. Circ Arrhythm Electrophysiol 2008; 1 (4): 258-68.

7. Bauer A, Malik M, Schmidt G, et al. Heart rate turbulence: standards of measurement, physiological interpretation, and clinical use: International Society for Holter and Noninvasive Electrophysiology Consensus. J Am Coll Cardiol 2008; 52 (17): 1353-65.

8. Hostetler B, Xue J, Young B, et al. Detect short run of TWA event with time-domain algorithm. Comput Cardiol 2005; 32: 483-6.

9. Mowatt G, Cook JA, Hillis GS, et al. 64-Slice computed tomography angiography in the diagnosis and assessment of coronary artery disease: systematic review and meta-analysis. Heart 2008; 94 (11): 1386-93.
10. Prati F. Utilizzo appropriato dell'angiografia coronarica con tomografia computerizzata nella cardiopatia ischemica: indicazioni e limiti [Coronary computed tomography angiography in coronary heart disease: clinical applications and limitations]. G Ital Cardiol (Rome) 2019; 20 (7): 409-16.

11. Веселовская Н.Г., Чумакова Г.А., Шенкова Н.Н., и др. Модель прогнозирования риска коронарного атеросклероза у пациентов с висцеральным ожирением. Рос. кардиол. журн. 2015; 4 (120): 49-54 [Veselovskaia NG, Chumakova GA, Shenkova NN, et al. Model' prognozirovaniia riska koronarnogo ateroskleroza u patsientov s vistseral'nym ozhireniem. Ros. kardiol. zhurn. 2015; 4 (120): 49-54 (in Russian)].

12. Котельникова Е.В., Гриднев В.И., Довгалевский П.Я., Беспятов А.Б. Прогнозирование коронарного атеросклероза для выбора тактики ведения больных ишемической болезнью сердца в амбулаторной практике. Кардиология. 2004; 44 (3): 15-9 [Kotel'nikova EV, Gridnev VI, Dovgalevskii Pla, Bespiatov AB. Prognozirovanie koronarnogo ateroskleroza dlia vybora taktiki vedeniia bol'nykh ishemicheskoi bolezn'iu serdtsa v ambulatornoi praktike. Kardiologiia. 2004; 44 (3): 15-9 (in Russian)].

13. Шарафеев А.З., Зыятдинов К.Ш., Акберов Р.Ф. Возможности ультразвукового ангиосканирования в раннем выявлении коронарного атеросклероза. Казан. мед. журн. 2011; 92 (4): 525-9 [Sharafeev AZ, Zyiatdinov KSh, Akberov RF. Vozmozhnosti ul'trazvukovogo angioskanirovaniia $v$ rannem vyiavlenii koronarnogo ateroskleroza. Kazan. med. zhurn. 2011; 92 (4): 525-9 (in Russian)]. 